\title{
Myocardial injury in patients after an elective abdominal aortic aneurysm repair
}

\author{
Mannova $\mathrm{J}^{1}$, Silhart $\mathrm{Z}^{2}$, Prokes $\mathrm{A}^{3}$, Sevcik $\mathrm{P}^{4}$ \\ Masaryk University, Faculty Hospital Brno, Czech Republic. j.mann@seznam.cz
}

\begin{abstract}
Background: The aim of the study was to study the frequency of cTnl release in patients undergoing an elective abdominal aortic aneurysm repair (AAA) and examine the relationship between an early perioperative myocardial injury, as detected by an increased serum levels of cTnl, and a clinical outcome (postoperative cardiac and non-cardiac complications, mortality).

Methods: A prospective observational study of 90 patients undergoing an elective AAA repair. Setting: University hospital. Blood samples were obtained from 90 patients for cTnl assays, 8 and 24 hours after surgery.

Results: $74.5 \%$ of patients faced a high and medium perioperative risk (ASA III-IV). Peak postoperative cTnI concentrations above the lower detection limit of the immunoassay occurred in 22 patients. 15 of these patients (cTnl levels: $0.2-10.2 \mathrm{ng} / \mathrm{ml}$ ) displayed symptoms of clinically significant complications. 9 patients (cTnl levels: $0.48-10.2 \mathrm{ng} / \mathrm{ml}$ ) had serious complications ( $3 x \mathrm{Ml}, 3 x$ heart failure, $2 x$ bronchopneumonia - respiratory failure, $1 \mathrm{x}$ sepsis), one patient died. Only $5.8 \%$ of patients without cTnl increase had postoperative complications. A Chi-square analysis revealed a significant association between cTi elevation and postoperative complications (chi-square 38.737, $\mathrm{p}<0.0001$ ).

Conclusions: A quarter of the patients who underwent an elective aortic surgery suffered from myocardial necrosis. A cTnl rise was associated with clinically significant events in $68 \%$; patients with cTnl release were at a significantly higher risk of cardiac and non-cardiac complications. These data confirm that patients with a postoperative rise in cTnl levels would be classified as patients at a higher risk of complications and a specific cardioprotective treatment, invasive monitoring and a longer ICU stay could be required in these cases (Tab. 6, Ref. 24). Full Text in PDF www.elis.sk.

Key words: heart, ischemia, surgery, aneurysm, complications, troponin.
\end{abstract}

An elective abdominal aortic aneurysm (AAA) repair is associated with a higher rate of perioperative and postoperative mortality and morbidity. The most frequent postoperative complications, which can lead to an increased postoperative mortality, are cardiac complications (15.1\%): myocardial infarction (MI) and heart failure $(1,2)$. The current mortality rate after the repair of the elective AAA remains about 1-7 \% (1). Perioperative myocardial infarction (MI) accounts for more than $50 \%$ of all deaths occurring after the elective aortic surgery (3).

It has been shown that cardiac troponins are highly sensitive and specific markers of myocardial injury (4-10). Studies have been already published showing the relationship between cTnI elevation and postoperative cardiac complications and mortality $(3,4,7,9,10)$. However, there is no study including only patients after an elective aortic abdominal aneurysm repair and investigating the association of cTnI increase with both cardiac and non-cardiac complications.

${ }^{1}$ Masaryk University, Faculty Hospital Brno, Czech Republic, ${ }^{2}$ Department of Surgery, Masaryk University, Faculty Hospital Brno, Czech Republic, ${ }^{3}$ Faculty of Electrical Engineering and Communication, Brno University of Technology, Czech Republic, and ${ }^{4} \mathrm{~F}$ aculty Hospital Ostrava, University Ostrava, Czech Republic

Address for correspondence: JH Mannova, PhD, Polni 17, CZ-591 02 Zdar nad Sazavou 2, Czech Republic.

Phone: +420.604232770
Abdominal aortic surgery is often performed in elderly patients who suffer from multiple comorbidities. During the operation, it is necessary to take into account haemodynamic changes, caused by aortic cross-clamping and declamping, and larger blood loss. We can probably assume that an increase in troponin I and frequency of postoperative complications would be higher or more evident in this patient population due to more severe trauma and ischemiareperfusion injury associated with this surgical intervention. In the view of the above-mentioned facts, the question is how frequent is myocardial injury in patients with the elective AAA, whether an increase in $\mathrm{cTnI}$ in the elective AAA surgery has a prognostic value and patients with $\mathrm{cTnI}$ increase have a significantly higher risk of postoperative complications.

The aim of this study was to investigate the frequency of cTnI release in patients undergoing the elective AAA repair and examine the relationship between an early perioperative myocardial injury, as detected by an increased serum levels of cTnI, and a clinical outcome (postoperative cardiac and non-cardiac complications, mortality).

\section{Patients and methods}

Ninety consentient patients undergoing elective abdominal aortic aneurysm repair were included into a prospective observa- 
tional study. Data were collected on the patient's age, sex, history of diseases and risk factors. Local research ethics committee's approval was obtained for this study.

All operations were performed under a general anaesthesia using opioids, benzodiazepines, muscle relaxants and volatile anaesthetics. Standard monitoring of heart rate, invasive arterial pressure, central venous pressure and urine output was used. All patients had an enhanced haemodynamic monitoring in the perioperative period, echo-oesophageal Doppler or pulmonary artery thermodilution. As a method of blood care, an acute normovolemic haemodilution or cell saver was used. All elective patients received an intravenous bolus of heparin before aortic clamping. No patients required suprarenal clamping.

Blood samples were obtained from all 90 patients for $\mathrm{cTnI}$ assays (immunoassay MAYO), 8 and 24 hours after surgery. The lower detection limit of this immunoassay was $0.1 \mathrm{ng} / \mathrm{ml}$ and a value equal to or higher than this level was considered positive for myocardial injury.

During perioperative period, the frequency of hypotension $(\mathrm{MAP}<70)$ and hypertension $(\mathrm{BP}>180 / 95, \mathrm{MAP}>125)$ was monitored, the same as the rate of serious haemodynamic complications: acute heart failure, ST changes $>1 \mathrm{~mm}$, new ventricular and supraventricular arrhythmias. The medians of length of operation and perioperative blood loss were calculated.

Symptoms and signs of postoperative complications were actively sought and recorded ten days after operation. These included cardiovascular complications: ECG and clinical evidence of MI, new or worsened angina pectoris, heart failure, cardiac rhythm changes, hypertension; and non-cardiac complications: pulmonary, renal, infectious and cerebrovascular complications, postoperative bleeding, coagulopathy and surgical complications. All in-hospital deaths and 30 days mortality were recorded, too.

The relationship between cTnI release and the frequency of serious complications was examined in the group of 90 patients. The Chi-square test was used for a statistical analysis.

\section{Results}

In a group of ninety patients, who were admitted with abdominal aortic aneurysm and underwent the open AAA repair, there were 71 men and 19 women of median age 72 (range 53-88 years). $74.5 \%$ of patients were at high or medium perioperative risk: ASA III-IV; the most frequent diseases in patient histories were MI (30\%), coronary artery disease (53\%) chronic heart failure (5.5\%), chronic valvular disease $(7.7 \%)$, chronic obstructive pulmonary disease-COPD (24\%), chronic renal insufficiency-CHRI (32\%), diabetes mellitus (18\%), and hypertension (61\%). Only $25.5 \%$ of patients were at a lower perioperative risk: ASA I-II (Tab. 1).

Perioperative hypotension or hypertension was displayed in 57 patients, perioperative heart failure in $5.6 \%$, ST changes $>1 \mathrm{~mm}$ in $3.3 \%$, and serious haemodynamic complications (heart failure, ST changes, new haemodynamic important arrhythmias) in $8.9 \%$ (Tab.2).

Cardiac postoperative complications developed in 13 patients, 3 of them had an acute MI, three congestive heart failure, one non stable angina pectoris, and 6 new arrhythmias. Pulmonary complications occurred in four patients, impairment of chronic renal
Tab. 1. Patients demographics, risk factors.

\begin{tabular}{lc}
\hline AGE & $\mathrm{n}(90)$ \\
\hline Age (median) & 72 \\
Age $>70$ years & 48 \\
GENDER & \\
Male & 71 \\
Female & 19 \\
\hline RISK FACTORS & 27 \\
\hline Myocardial infarction (MI) & 18 \\
Angina pectoris II- III degree & 48 \\
Coronary artery disease & 5 \\
Chronic Heart Failure & 14 \\
Ejection fraction (EF) <35\% & 7 \\
Serious valvular disease & 2 \\
Pulmonary emboli & 12 \\
Supraventricular arrhythmias, AV block & 2 \\
Ventricular arrhythmias & 16 \\
Diabetes mellitus & 55 \\
Hypertension & 29 \\
Chronic renal insufficiency (CHRI), creatinine $>110$ umol/l & 22 \\
Smoking. > 20 cigarette/ Chronic obstructive pulmonary disease & \\
(COPD). FEV/FVC 0.6-0.45 & 14 \\
Stroke & 12 \\
Obesity & \\
\hline CARDIOVASCULAR RISK & 67 \\
\hline High and medium cardiovascular (CV) risk, ASA III-IV \\
Low CV risk, ASA I-II & 23 \\
Size of abdominal aneurysm mm (average) & 69 \\
\hline
\end{tabular}

Tab. 2. Perioperative complications, length of operations, perioperative blood loss.

\begin{tabular}{lc}
\hline Perioperative complications $\mathrm{n}=90$ & $\mathrm{n}$ \\
\hline Hypotension $(\mathrm{MAP}<70)$ & 42 \\
Hypertension $(\mathrm{TK}>180 / 95, \mathrm{MAP}>125)$ & 17 \\
Acute heart failure & 5 \\
Ventricular arrhythmias & 7 \\
ST- changes $>1 \mathrm{~mm}$ & 3 \\
Serious hemodynamic complications & 8 \\
Length of operations (median) & $200 \mathrm{~min}$ \\
Perioperative blood loss (median) & $971 \mathrm{ml}$ \\
\hline
\end{tabular}

failure in 9 patients and none of them had an acute renal failure requiring dialysis. Mortality in the group of 90 patients was $1.1 \%$, median of lengths of ICU stay were 4 days, and the median of overall hospital stay was 10 days. All postoperative complications are shown in Table 3.

Peak postoperative cTnI concentrations above the lower detection limit of the immunoassay $(0.1 \mathrm{ng} / \mathrm{ml})$ occurred in $22 \mathrm{pa}-$ tients. In the group of patients with $\mathrm{cTnI}$ increase, sixteen patients ( $73 \%$ ) were ASA III-IV and six patients (27\%) were ASA II; by comparison, 51 patients $(75 \%)$ without $\mathrm{cTnI}$ increase were ASA III-IV and 17 patients ( $25 \%$ ) were ASA II (Tab. 4). These figures show that both groups of patients at lower and higher perioperative risk had an increase in cTnI. When the frequency of COPD and CHRI in the patients ' histories was compared, chronic renal insufficiency (creatinine $>110 \mathrm{umol} / \mathrm{l}$ ) was more frequent in patients with positive cTnI levels than in patients without positive cTnI levels (50\% vs $26 \%$ ); a similar result was gained for chronic obstructive disease (FEV/FVC 0.6-0.45), which was more frequent in patients with increased cTnI levels ( $32 \%$ vs $22 \%$ ) too. 
Tab. 3. Postoperative complications.

\begin{tabular}{lcc}
\hline Postoperative complications $\mathrm{n}=90$ & $\mathrm{n}$ & $\%$ \\
\hline Haemodynamic complications & & \\
Acute myocardial infarction (AMI) & 3 & $3.30 \%$ \\
Chest pain - non stabile angina pectoris (NAP) & 1 & $1.10 \%$ \\
Congestive heart failure (CHF) & 3 & $3.30 \%$ \\
New arrhythmias (atrial fibrillation) & 4 & $4.40 \%$ \\
Pulmonary complications & & \\
Bronchopneumonia with respiratory failure & 2 & $2.20 \%$ \\
Bronchopneumonia & 2 & $2.20 \%$ \\
Pulmonary embolism & 0 & $0 \%$ \\
Renal complications & & \\
Acute renal failure (ARF) & 0 & $0 \%$ \\
Infection, Sepsis, multi organ failure & 1 & $1.10 \%$ \\
Haemorrhagic shock & 0 & $0 \%$ \\
Disseminated Intravascular Coagulopathy - DIC & 1 & $1.10 \%$ \\
Transient ischemic attack - TIA & 2 & $2.20 \%$ \\
Surgical complications & & \\
Paralytic ileus & 4 & $4.40 \%$ \\
Dehiscention & 2 & $2.20 \%$ \\
Reoperation & 1 & $1.10 \%$ \\
Limb ischemia - graft thrombosis & 2 & $2.20 \%$ \\
Paraplegia & 1 & $1.10 \%$ \\
Mortality & 1 & $1.10 \%$ \\
\hline
\end{tabular}

Tab. 4. Comparison of groups with $\mathrm{cTnI}$ increase $(\mathrm{cTnI}+)$ and without cTnI increase (cTnI-).

\begin{tabular}{lcccc}
\hline & $\begin{array}{c}\mathrm{cTnI}+ \\
\mathrm{n}=22\end{array}$ & $\%$ & $\mathrm{cTnI}-$ & $\%$ \\
& 16 & $73 \%$ & 51 & $75 \%$ \\
\hline ASA III-IV & 6 & $27 \%$ & 17 & $25 \%$ \\
ASA I-II & 7 & $32 \%$ & 15 & $22 \%$ \\
Chronic obstructive pulmonary disease & 11 & $50 \%$ & 18 & $26 \%$ \\
Chronic renal insufficiency & 2 & $9 \%$ & 6 & $9 \%$ \\
Perioperative depression of the ST & & & & \\
segment, heart failure & 15 & $68 \%$ & 4 & $5.80 \%$ \\
Postoperative complications & 8 & $36 \%$ & 3 & $4.40 \%$ \\
Postoperative cardiac complications & 7 & $32 \%$ & 1 & $1.40 \%$ \\
Postoperative non-cardiac complications & 1 & $4.50 \%$ & 0 & $0 \%$ \\
Mortality & & & & \\
\hline
\end{tabular}

If the incidence of serious perioperative complications was compared, the frequency of perioperative heart failure and ST depression was the same in the group with $\mathrm{cTnI}$ increase as in the group without $\mathrm{cTnI}$ increase (9\% vs $9 \%$ ).

In the terms of postoperative complications (Tab. 4), $68 \%$ of patients with $\mathrm{cTnI}$ increase developed postoperative complications in contrast to $5.8 \%$ of patients without cTnI increase, similarly cardiac and non-cardiac complications occurred more frequently in patients with cTnI increase (cardiac: $36 \%$ vs $4.4 \%$; non-cardiac $32 \%$ vs $1.4 \%$ ). One predisposed patient (COPD) died of postoperative bronchopneumonia with respiratory failure in a group of patients with positive $\mathrm{cTnI}$ levels in contrast to no death in a group with negative cTnI levels (mortality: $4.5 \%$ vs $0 \%$ ).

From twenty two patients $(24.4 \%)$, who had a detectable level of cTnI at least once during the first $48 \mathrm{~h}$ after operation, fifteen patients (cTnI levels: $0.2-10.2 \mathrm{ng} / \mathrm{ml}$ ) displayed symptoms of clinically significant complications (Tab. 5). Eight patients had cardiac complications: MI, heart failure, new arrhythmias; median cTnI levels: 1.99 (range 0.4-10.2) ng/ml and seven patients had non cardiac complication: bronchopneumonia, sep-
Tab. 5. Levels of cTnI.

\begin{tabular}{lc}
\hline Cardiac complications & cTnI levels ng/ml \\
\hline Atrial fibrillation & 0.7 \\
Acute myocardial infarction & 10.2 \\
Acute heart failure & 4.9 \\
Acute heart failure & 0.48 \\
Acute myocardial infarction & 3.2 \\
Acute myocardial infarction & 5.5 \\
Chest pain, NAP & 0.78 \\
Acute heart failure & 0.4 \\
& median 1.99 \\
\hline Non-cardiac complications & \\
\hline TIA & 1.8 \\
Sepsis with multi organ dysfunction & 0.4 \\
Bronchopneumonia with respiratory failure & 0.3 \\
TIA & 0.3 \\
Bronchopneumonia with respiratory failure & 4.6 \\
Bronchopneumonia & 0.2 \\
DIC & 0.4 \\
& median 0.4 \\
\hline
\end{tabular}

Tab. 6. Chi-square analysis between cTnI elevations and postoperative complications.

\begin{tabular}{lcccc}
\hline & & \multicolumn{3}{c}{ Postoperative complications } \\
\cline { 2 - 5 } & & yes & no & total \\
\hline cTnI elevation & yes & 15 & 7 & 22 \\
& no & 4 & 64 & 68 \\
& total & 19 & 71 & 90 \\
\hline
\end{tabular}

$\left(\chi^{2}(1, \mathrm{n}=90)=38.737 ; \mathrm{p}<0.0001\right)$

sis, transitory ischemic attack (TIA), disseminated intravascular coagulopathy; median cTnI levels: 0.4 (range $0.2-4.6$ ) ng/ml. Patients with cardiac complications had higher cTnI levels than those with non-cardiac complications (Tab. 5). Nine of the 15 patients with cTnI increase had serious complications (cTnI levels: 0.4-10.2 ng/ml). Six complications were cardiac (MI, heart failure), three patients had myocardial infarction with clinical and electrocardiographic evidence. Heart failure with the evidence of an acute cardiac dysfunction, revealed by echocardiography, appeared in three of these patients. Three patients had non-cardiac complications - two of them bronchopneumonia with respiratory failure and one had sepsis with multiple organ dysfunction.

The remaining seven patients with a raised cTnI level did not have any clinically apparent cardiac or non-cardiac events. These patients did not have significantly lower median cTnI levels than those with clinically evident complications: 0.5 (range: $0.2-3.8$ ) versus $0.7(0.2-10.2) \mathrm{ng} / \mathrm{ml}$.

Out of nineteen patients who developed postoperative complications, fifteen patients had elevated levels of troponin I, which predated the appearance of clinical symptoms. Only four patients had postoperative complications without increased in cTnI levels, new arrhythmias (atrial fibrillation) were recorded in three of these patients and one patient developed bronchopneumonia. All clinically significant complications (myocardial infarction, heart failure, sepsis, transient ischemic attack and bronchopneumonia) were associated with raised cTnI levels.

When the relationship between postoperative complications and cTnI increase was investigated, a Chi square analysis revealed 
a significant association between cTi elevation and postoperative complications. Patients with $\mathrm{cTnI}$ release were at a significantly higher risk of postoperative complications with $p<0.0001$ (Tab. 6).

\section{Discussion}

The main finding of the study is that $24.4 \%$ of patients had myocardial damage detected by an increased serum levels of cTnI after the elective abdominal aortic aneurysm surgery. Patients with cTnI increase had a significantly higher risk of postoperative complications and 15 (68\%) of the 22 patients with cTnI increase developed postoperative complications, both cardiac and non-cardiac.

By comparison, the incidence of cTnI elevation in Tambyraja's study (9) was twice as high as in our study. Tambyraja et al investigated 62 patients who underwent operative repair of a ruptured AAA. $81 \%$ of these patients survived for more than 24 hours, twenty-three patients ( $46 \%$ ) had a detectable cTnI level during the first 48 hour. Of these, 11 patients $(48 \%)$ had clinical or ECG evidence of an acute cardiac event and $12(52 \%)$ did not. Ten patients with cTnI increased and three without $\mathrm{cTnI}$ increased died within 30 days after operation. In our group of patients after the elective AAA operations, only one patient died and the frequency of cardiac injury was approximately a half lower $(24.4 \%)$. We can assume that patients with a ruptured AAA are subject to a greater risk of perioperative cardiac injury. This is likely due to the impact of massive haemorrhage and transfusion. In the group of patients with the ruptured AAA in Tambyraja's study, the median of blood loss was 3000 (1325-8500) $\mathrm{ml}$ (cTnI negative) and $4600 \mathrm{ml}$ (1070-21000) $\mathrm{ml}$ (cTnI positive) in contrast to patients after the elective operative repair of AAA in our study, where the median of blood loss was only $971(300-3500) \mathrm{ml}$. In case of critically ill patients in the intensive care, the frequency of myocardial injury was reported between 15 to $40(474+395 / 2591) \%(11-13)$. If we compare the incidence of myocardial injury in patients who undergo major vascular operation (aortic and carotid surgery), our findings are in agreement with the results of Landesberg's and Haggart's studies $(3,14)$. Landesberg et al (14) described that $23.9 \%$ of vascular surgery patients developed perioperative myocardial injury determined by raised levels of cardiac troponins. Haggart et al (3) published that more than a quarter of patients having the elective aortic surgery had myocardial damage.

The elevations of troponin, which we observed, were mostly small to modest. In our group of 90 patients, only six patients had cTnI levels above $2 \mathrm{ng} / \mathrm{ml}$, the rest of patients had troponin I 0.2 $2.0 \mathrm{ng} / \mathrm{ml}$; median $0.55 \mathrm{ng} / \mathrm{ml}$. Although majority of our patients had moderate or minor cTnI levels, it was published in previous studies that moderate elevations of serum troponin I, which are below the threshold required to diagnose overt myocardial infarction, may reflect ongoing myocardial injury and are associated with longer ICU and hospital stay $(9,12)$. It has been demonstrated that a slight, clinically silent increase in the cTnI level within the first $48 \mathrm{~h}$ after operation meant an increased risk of postoperative death (12). According to the study carried out by Relos et al (12) patients with moderate and minor elevations of serum troponin I (0.4-2.0 ug/l) had a significantly higher mortality rate (chi-square $32.57, \mathrm{p}<0.0001)$ and longer stays in the intensive care unit and hospital $(\mathrm{p}<0.0005)$ when compared to patients without similar elevations. Similar results were obtained in Tambyraja's study (9).

In our study, increased levels of cTnI predated both cardiologic events (MI, heart failure, new arrhythmias) and non-cardiologic postoperative complications (infection, disseminated intravascular coagulopathy, sepsis, TIA). The contribution of our study is that the incidence of all kind of postoperative complications, not only cardiac events, was recorded, contrary to other studies (3, 4, $10,14)$ in vascular surgery, which investigated only association between postoperative cardiac complications and $\mathrm{cTnI}$ release. In published studies, the increase in cTnI was linked to ischemic myocardial damage and due to this; the incidence of postoperative MI or postoperative cardiac complications was studied.

As shown in our study, the increase in cTnI can predict besides cardiac complications also non-cardiac complications, because several mechanisms can lead to raised troponins. The bestknown reason for the increase in $\mathrm{cTnI}$ is myocardial ischemia in the setting of acute coronary syndromes (MI) (7). However, other mechanisms may be relevant, too (15). For example, toxic substances can directly produce cardiac injury (16). Cardiac damage can be ischemic but not due to overt epicardial coronary artery disease (8). Possible etiologies for cardiac injury in the absence of coronary artery disease include subendocardial injury caused by increased wall stress as occurs in congestive heart failure (17); hypertension with left ventricular hypertrophy leading to supplydemand imbalances; increased myocardial oxygen consumption or increments in right ventricular afterload in patients with acute pulmonary embolism (18). In our study, cTnI elevation predated sepsis and disseminated intravascular coagulopathy. Toxic insult in sepsis may exacerbate underlying ischemic heart disease or could function to damage the heart directly (8). Elevation can be due to the effects of cytokines such as heat shock protein and tumor necrosis factor (7-9). Tumour necrosis factor has been shown to increase the permeability of endothelial monolayers, leading to leakage of cardiac troponins (19). A further mechanism for a rise in troponins in sepsis may be related to the coagulation in the capillary bed, leading to reversible myocardial hypoxia and apoptosis associated with a leakage of cardiac troponins into the serum (7). A relation has been found to exist between increased troponin levels and mortality in patients with sepsis $(13,20)$. Additional cardiac damage can be secondary to inflammation (myocarditis) or an infiltrative process that involves the myocardium (8). Four patients in our study had increased level in cTnI before the development of bronchopneumonia with or without acute respiratory failure, and another two patients before TIA. The elevation of troponin in patients with acute respiratory failure could be due to concomitant coronary artery disease, heart failure or acute pulmonary process that can increase the left or right ventricular wall stress (15). Troponin elevations in patients with acute ischemic stroke have been reported too, clinical trial data have incessantly indicated that neurologically induced myocardial injury exists. Because various electrocardiographic alterations have also been found in these patients, it has been suggested that elevated troponin levels are somehow neurologically mediated, thus not caused by direct cardiac release (21). No patients in our study had pulmonary embolism as 
a postoperative complication but we can assume that cTnI elevation would predate this complication too. Acute right ventricular strain secondary to increases in pulmonary arterial resistance can be the cause of troponin elevations in pulmonary embolism. Moreover, serum troponin levels can help stratify patients with submassive acute pulmonary embolism into a group in which aggressive medical or surgical intervention would be considered $(22,23)$.

Patients with cTnI release in our study had a significantly higher risk of postoperative complications $(\mathrm{p}<0.0001)$ which were recorded in $68 \%$ of patients with cTnI elevation. Of additional importance is that if elevations of troponin can identify patients with the risk of postoperative complications, there may be an opportunity to intervene in these patients. The finding of a cTnI rise should lead us to put the patient with increased $\mathrm{cTnI}$ in a higher risk category. This knowledge could prompt changes in invasive monitoring and the commencement of protective therapy. A variety of potential therapies exists, including beta blockers, low molecular weight heparins, antiplatelet receptor antagonists and even early coronary revascularization (3, 24). These treatments may have a similar important role in reducing morbidity and mortality rates from perioperative microinfarction.

In conclusion, almost a quarter of patients in our study undergoing the elective aortic surgery suffered from myocardial necrosis as determined by detectable cTnI levels. The cTnI rise was associated with clinically significant events in $68 \%$ and all serious postoperative complications were predated by an increase in cTnI concentration $(0.4-10.2 \mathrm{ng} / \mathrm{ml})$. In the group of patients without cTnI increase only $5.8 \%$ of patients had postoperative complications. Patients with positive cTnI levels were at a higher risk of postoperative cardiac and non-cardiac complications, and the Chi square analysis revealed a significant association between cTi elevation and occurrence of postoperative complications. These data suggest that patients with a postoperative rise in cTnI levels would be classified as patients at a higher risk for postoperative complications. Invasive monitoring, longer ICU stays and a specific cardioprotective treatment could be required in these cases.

\section{References}

1. Ghansan JN, Murphy T. Complications of Major Aortic and Lower Extremity Vascular Surgery. Semin Cardiothorac Vasc Anesth 2004; 8: 335-361.

2. Johnston KW. Multicenter prospective study of nonruptured abdominal aortic aneurysm. Part II Variables predicting morbidity and mortality. J Vasc Surg 1989; 9: 437-447.

3. Haggart PC, Adam DJ, Ludman PF, Bradbury AW. Comparison of cardiac troponin I and creatine kinase ratios in the detection of myocardial injury after aortic surgery. Br J Surg 2001; 88: 1196-1200.

4. Andrews N, Jenkins J, Andrews G, Walker P. Using postoperative cardiac troponin I (cTnI) levels to detect myocardial ischemia in patients undergoing vascular surgery. Cardiovasc Surg 2001; 9: 254-265.

5. Katus H, Remppis A, Scheffold T, Dietrich KV, Kubler W. Intracellular compartmentation of cardiac troponin $\mathrm{T}$ and its release kinetics in patients with reperfused and nonreperfused myocardial infarction. J Am Cardiol 1992; 67: 1360-1364.

6. Morrow DA, Cannon CP, Rifai N, Frey MJ, Vicari R et al. Ability of Minor Elevations of Troponins I and T to Predict Benefit from an Early
Invasive Strategy in Patients with Unstable Angina and non ST-Elevation Myocardial Infarction. JAMA 2001; 19: 2405-2412.

7. Ammann P, Pfisterer M, Fehr T, Rickli H. Raised cardiac troponins. BMJ 2004; 328: 1028-1029.

8. Babuin L, Jaffe AS. Troponin: the biomarker of choice for the detection of cardiac injury. CMAJ 2005; 173: 1191-1197.

9. Tambyraja AI, Dawson AR, Murie JA, Chalmers TA. Cardiac troponin I predicts outcome after ruptured abdominal aortic aneurysm repair. Br J Surg 2005; 92: 824-827.

10. Howell SJ, Thompson JP, Nimmo AF, Snowden C, Edwards ND et al. Relationship between perioperative troponin elevation and other indicators of myocardial injury in vascular surgery patients. BJA 2006, 96: 303-309.

11. Guest TM, Ramanathan AV, Tuteur PG, Schechtman KB, Landenson JH, et al. Myocardial injury in critically ill patients. A frequently unrecognized complication. JAMA 1995; 273: 1945-1949.

12. Relos RP, Hasinoff IK, Beilman GJ. Moderately elevated serum troponin concentrations are associated with increased morbidity and mortality rates in surgical intensive care unit patients. Crit Care Med 2003; 31: 2598-2603.

13. Kollef MH, Laenson JH, Eisenber PR. Clinically recognized cardiac dysfunction: an independent determinant of mortality among critically ill patients. Is there a role for serial measurement of cardiac troponin I? Chest 1997; 111: 1340-1347.

14. Landesberg G, Shatz V, Akopnik I, Wolf JG, Mayer M et al. Association of Cardiac Troponin, CK-MB, and Postoperative Myocardial Ischemia with Long-Term Survival After Major Vascular Surgery. J Am Cardiol 2003; 42: 1547-1554.

15. Babuin L, Vasile VC, Rio Perey JA, Alegria JR, Chai H et al. Elevated cardiac troponin is an independent risk factor for short and long-term mortality in medical intensive care unit patients. Crit Care Med 2008; 36: 759-765.

16. Wu AH. Increased troponin in patients with sepsis and septic shock: Myocardial necrosis or reversible myocardial depression? Intensive Care Med 2001; 27: 959-961.

17. Potluri S, Ventura HO, Mulumundi M, Mehra MR. Cardiac troponin levels in heart failure. Cardiology in review 2004; 12: 21-25.

18. De Genaro L, Brunetti ND, Cuculo A, Pellegriono PL, Izzo P et al. Increased troponin levels in nonischemic cardiac conditions and noncardiac diseases. J Intervent Cardiol 2008; 21: 129-139.

19. Brett J, Gerlach H, Nawroth P, Steinberg S, Godman G et al. Tumor necrosis factor/cachectin increases permeability of endothelial cell monolayers by a mechanism involving regulatory G proteins. J Exp Med 1989; 169: 1977-1991.

20. Spies C, Haude V, Fitzner R. Serum cardiac troponin T as a prognostic marker in early sepsis. Chest 1998; 113: 1055-1063.

21. Jenssen JK, Atar D., Mikley H. Mechanism of troponin elevations in patients with acute ischemic stroke. Am J of Cardiol 2007; 99: 867-870.

22. Konstantinides S, Geibel A, Olschewski M. Importance of cardiac troponins I and $\mathrm{T}$ in risk stratification of patients with acute pulmonary embolism. Circulation 2002; 106: 1263-1268.

23. Horlander KT, Leeper KV. Troponin levels as a guide to treatment of pulmonary embolism. Curr Opin Pulm Med 2003; 9: 374-377.

24. Yeager FA, Moneta GL, Edwards JM, Taylor LMJ, BcConnell DB et al. Reducing perioperative myocardial infarction following vascular surgery. The potentioal role of beta-blockade. Arch Surg 1995; 130: 869-887.

Received February 11, 2011. Accepted January 18, 2013. 